\title{
REFLEXIONES DE UNA PROFESORA DE SECUNDARIA SOBRE LA ENSEÑANZA DE LA FILOSOFÍA
}

\section{Clara Inés Jaramillo Gaviria}

\section{INTRODUCCIÓN}

Al inscribirme en el curso de "Enseñanza de la filosofía", ofrecido por la Universidad Nacional de Colombia ${ }^{1}$, sentí una gran motivación, pues es poca la formación que ofrecen las Universidades a los docentes de secundaria del área de filosofía. Si bien se dan cursos especializados en temas filosóficos o en filósofos, son pocos los cursos que se centran en la enseñanza de la filosofía como asignatura, y que trabajan su didáctica, metodología, currículo, etc. Este curso prometía llenar ese vacío, ya que afirmaba tener como fin: "Contribuir a la formación docente en el área de la filosofía, reflexionando acerca de su enseñanza en la educación secundaria" .

El programa del curso anunciaba abordar la temática en dos módulos: uno histórico, cuyo objetivo era acercarse a las concepciones sobre pedagogía de algunos de los filósofos más representativos en la historia del pensamiento; y otro metodológico, que versaría sobre los problemas centrales de la enseñanza de la disciplina, el manejo de conceptos básicos y el uso de metodologías ${ }^{3}$.

Si bien el programa planteado era atractivo, consideramos que su desarrollo no llenó las expectativas, pues, una vez más, la discusión se centró en cuestiones teóricas y en situaciones ideales, dejando de lado las realidades concretas a las que nos vemos enfrentados diariamente los profesores de secundaria. Los conferencistas, docentes universitarios, centraron más su mirada en lo que los filósofos dicen sobre la enseñanza de la filosofía, -teorías sobre el aprender, diferentes métodos etc-, olvidando el centro de la discusión: ¿Cómo enseñar filosofía en secundaria? ¿Qué estrategias pedagógicas se deben implementar

\footnotetext{
${ }^{1}$ Departamento de Filosofía. Curso de Extensión: ENSEÑANZA DE LA FILOSOFÍA. Universidad Nacional de Colombia, septiembre 20 - Diciembre 6 de 2005.

${ }^{2}$ Ibíd. (Programa) p. 1.

${ }^{3}$ Ibídem.
} 
para enseñar filosofía a jóvenes que no manejan conceptos filosóficos, léxico, autores, épocas, etc.? ¿Cómo hacer atractiva la enseñanza de la filosofía en secundaría? $Y$ algo más profundo ¿para qué le sirve la filosofía a un joven bachiller? Estos son los interrogantes que nos hacemos constantemente los docentes de secundaria, y sobre los cuales pedimos, a la comunidad de filósofos, nos ayuden a dar respuesta.

Lo que si nos quedó claro, no sólo al concluir este curso, sino al escuchar muchas conferencias sobre educación, metodología, didáctica, etc., es que hay una brecha entre la teoría y la praxis educativa. Los teóricos parten de un estudiante "ideal", aulas "ideales", y condiciones de aprendizaje "óptimas". En cambio los docentes de secundaria nos encontramos con realidades que, en su gran mayoría, rompen con el ideal: cursos de más o menos 45 estudiantes; jóvenes permeados por la tecnología y el consumismo, desilusionados del sistema educativo y alejados de las reflexiones filosóficas; jóvenes que viven el día a día sin pensar en el pasado o en el futuro, en un mundo hedonista, y que asisten al colegio más por obligación que por el deseo de aprender. Son pocos los que realmente le dan sentido y proyección a su formación personal e intelectual. ¿No es desde aquí de donde debe partir la discusión?

En este contexto, y con el fin de encontrar caminos, quiero proponer a la comunidad de docentes, tanto universitarios como de secundaria, que reflexionemos en torno a los siguientes cuestionamientos: 1. ¿Cuáles son las directrices dadas en Colombia por el Ministerio de Educación Nacional (MEN)? 2. ¿Qué se enseña actualmente en Colombia en la asignatura de filosofía? 3. ¿Qué nos queda por hacer?

\section{¿Cuáles son las Directrices Dadas en Colombia por el Ministerio de Educación Nacional?}

Antes de referirnos a lo que el Ministerio de Educación Nacional estipula para la enseñanza de la filosofía en secundaria, consideramos pertinente describir 
algunas directrices generales de este organismo que, de manera indirecta, repercuten en el diseño curricular de la asignatura de filosofía.

\section{Ley General de Educación: Ley 115 de 1994}

Desde la década de los noventa, -Ley 115, artículo 77- el Ministerio de Educación Nacional da inicio a una reforma que, entre otras cosas, pretende descentralizar la autoridad y dar cierta autonomía a las instituciones educativas regionales; implementar la elaboración de diseños curriculares autónomos, basados en las demandas y necesidades locales; y desarrollar estrategias organizacionales y pedagógicas que den respuesta a la problemática escolar y regional. Como consecuencia de lo anterior, las instituciones educativas comienzan a desarrollar sus propias estrategias, y a formular los principios sobre los cuales se fundamenta la educación de los estudiantes que a ellas pertenecen.

Según esto, el Ministerio de Educación Nacional -Decreto 1860 de Agosto 3 de 1994. Artículo 15- delega funciones y facultades de decisión a las instituciones, pero mantiene el control sobre la información y los recursos con el fin de orientar, evaluar y reajustar, en forma periódica, los procesos globales de gestión educativa. Para allanar este objetivo, el Ministerio de Educación Nacional exige a las instituciones educativas desarrollar un Proyecto Educativo Institucional. Éste debe enunciar las metas y objetivos que la institución ha de cumplir para la realización de su Misión Institucional, y de los fines formativos que se derivan de ella. Es decir, por medio del Proyecto Educativo Institucional la institución puede proyectar un plan de trabajo a mediano y largo plazo, pensarse en su dinámica histórica, prefigurar su posible futuro y explicitar su tarea social. La institución educativa, a la luz del Proyecto Educativo Institucional, debe desarrollar su propio currículo.

Para desarrollar el diseño curricular, la institución debe tener presente las necesidades sociales de la comunidad en la cual esté inserta, debido a que es en ésta donde se verá su impacto. Por eso, el currículo debe abordar, además de núcleos temáticos, núcleos problemáticos, pues la interacción de estos dos ejes 
permite relacionar los contenidos generales con los problemas e intereses específicos tanto del estudiante como de la comunidad ${ }^{4}$.

Las directrices de la Ley 115 se especifican y amplían en el documento Estándares básicos de Competencias. Éstos tienen como objetivo: Flexibilizar los diseños curriculares, y abrirse a las enormes posibilidades que ofrece el contexto natural y social para desarrollar procesos de formación con sentido y significado para los y las estudiantes ${ }^{5}$.

Los estándares básicos de competencias apuntan a los mínimos que el docente debe promover en los estudiantes para que sean personas sanas e íntegras; que participen activamente y de manera constructiva en su medio social y en el mundo; que interactúen con cuatro tipo de saberes: un saber conceptual, un saber procedimental (saber hacer), un saber actitudinal (saber ser) y un saber metacognitivo (saber aprender).

En consecuencia, el estudiante muestra ser competente cuando es capaz, por un lado, de poner el conocimiento conceptual, de forma eficaz y eficiente, a su servicio y al del medio en el que se desenvuelve; $y$, por otro lado, cuando es capaz de reflexionar, reproducir y mejorar su desempeño en nuevas situaciones o contextos. Por esta razón es que las competencias son concebidas como saberes de relevancia y pertinencia social y efectiva para los estudiantes. Esto es lo que hace de la educación un proceso de aprendizaje significativo (noción epistemológica que lidera la propuesta curricular vigente).

En este contexto cabe preguntar ¿dónde queda, dentro del modelo de educación propuesto por el Ministerio de Educación Nacional, el pensar crítico y/o divergente que caracteriza por excelencia a la actitud filosófica? ¿No es acaso tarea de la educación -además de capacitar personas eficaces, eficientes que reproducen 0 mejoran su desempeño en la sociedad-, formar personas creativas y originales

\footnotetext{
${ }^{4}$ Cfr. Ministerio de Educación Nacional. Decreto 230 de febrero 11 de 2002. Artículo 2.

5 Ministerio de Educación Nacional. Estándares Básicos de Competencias en Lenguaje, Matemáticas, Ciencias y Ciudadanía. Documento No. 3. Bogotá: M.E.N., 2006, p. 103.
} 
que propongan alternativas nuevas o distintas a las de la sociedad actual (como lo han hecho los filósofos que han transformado la historia humana)?

Una vez vistas las cuestiones generales que estipula el Ministerio de Educación Nacional veamos

\section{¿Qué lugar ocupa la enseñanza de la filosofía en el modelo educativo del MEN?}

Entrando ya en la temática que nos compete, de antemano tenemos que decir que no son claras las directrices que el Ministerio de Educación Nacional estipula para la enseñanza de la filosofía en secundaria, y que, como consecuencia de esta confusión, la asignatura se diluye en el currículo, ya sea como eje trasversal de formación, ya como parte de las ciencias sociales, ya como educación para la ciudadanía. Si bien la filosofía atañe a esto temas, consideramos que reducirla a ellos equivaldría a restringir, de manera significativa, la formación filosófica de los estudiantes. Veamos cómo se da este proceso.

La ley 115 establece unas áreas obligatorias y fundamentales, y entre ellas no se encuentra la filosofía ${ }^{6}$. De esta manera el Ministerio de Educación Nacional ya no reconoce la asignatura de filosofía como parte fundamental del currículo, ni siquiera en los grados superiores; y da autonomía a los directivos y docentes para que, a la luz del Proyecto Educativo Institucional, implementen su contenido y metodología, teniendo en cuenta el perfil de persona que desean formar.

Igualmente, la Ley 115 asumió que la formación en valores, actitudes éticas y competencias ciudadanas era el eje transversal que debía permear todo el currículo de la educación media, ya que permite entender los fundamentos de la democracia y desarrollar la capacidad cognitiva y afectiva exigidas en una sociedad compleja y cambiante como la nuestra: "Formación ética y moral. La formación ética y moral se promoverá en el establecimiento educativo a través del currículo, de los contenidos académicos pertinentes, del ambiente, del comportamiento honesto de directivos, educadores, y personal administrativo, de

\footnotetext{
${ }^{6}$ Congreso de la República. Ley General de Educación - Ley 115 de 1994. Artículo 23.
} 
la aplicación recta y justa de las normas de la institución, y demás mecanismos que contemple el Proyecto Educativo Institucional ${ }^{7 "}$.

Si bien es cierto que la ética y la axiología son disciplinas filosóficas, no podemos reducir su contenido sólo a estas dos áreas; hacerlo significaría tomar la parte por el todo. Además ¿qué parte del currículo tendría como fin hacer una reflexión académica pertinente sobre las temáticas éticas y morales? ¿Con qué parámetros los directivos, docentes y estudiantes evaluarían qué es una conducta honesta, recta, justa o conductas contrarias? Consideramos que en este sentido la filosofía, al reflexionar sobre los fundamentos de la moral y sobre sus distintas manifestaciones, sería un aporte desaprovechado si su contenido se diluye como un simple eje transversal del currículo.

Por otro lado, si confundimos el contenido de la filosofía con el de las ciencias sociales o ciencias humanas, volvemos a cometer el error de tomar la parte por el todo, ya que las ciencias sociales, por su propia naturaleza, desarrollan un conocimiento específico y especializado, mientras que la filosofía tiene como fin la construcción de un conocimiento universal. Además, la filosofía tiene también como objetivo reflexionar críticamente sobre los presupuestos de dichas ciencias y las posibles relaciones interdisciplinarias con otros tipos de saber, como el de las ciencias naturales, por ejemplo.

Podríamos decir que está apreciación es válida, pues, como afirma Salazar Bondy: "Cuando el hombre problematiza todos esos conocimientos adquiridos en la actitud científica, entra en la etapa de la actitud filosófica. Y busca los principios últimos, el fundamento y sentido del mundo, capaz de ofrecer una explicación satisfactoria del conjunto de la experiencia"8.

Sin embargo ¿cómo reflexionar sobre los fundamentos de las ciencias naturales y sociales al margen de la filosofía? ¿Cómo promover la actitud filosófica, si no se enseña filosofía y a filosofar? ¿Cómo aprender a filosofar, si no se entra en contacto directo con los grandes pensadores y sus teorías? ¿Cómo abordar los

\footnotetext{
${ }^{7}$ Congreso de la República. Ley General de Educación - Ley 115 de 1994. Artículo 25.

${ }^{8}$ Miró Quesada, F.; Salazar Bondy, A. Manual de filosofía. Volumen II. Lima, Universo, 1961, p. 40.
} 
problemas filosóficos sin tener en cuenta las perspectivas que sobre ellos abre el desenvolvimiento de las teorías, las escuelas y las corrientes del pensamiento universal?

\section{Otra de las dificultades a las que se enfrenta la enseñanza de la filosofía en secundaria surge cuando comparamos la Legislación del Ministerio de Educación Nacional y la Evaluación del Examen de Estado del ICFES.}

Una situación paradójica resulta al analizar los Estándares Básicos de Competencias en Ciencias Sociales y cotejarlos con los logros que pretende evaluar el examen del estado -ICFES-

"La evaluación por competencias en filosofía: La evaluación de la filosofía como un saber-hacer en contexto implica hacer explícitos los ámbitos específicos en que se han desarrollado los interrogantes y concepciones fundamentales de la filosofía... Los ámbitos que se asumen en la evaluación de la filosofía -epistemológico, antropológico, ético, estético y ontológico- tienen que ver con amplios campos del conocimiento filosófico que han sido objeto de reflexión, tanto en el transcurso de la historia de la filosofía, como en el contexto de la educación filosófica escolar. Estos campos filosóficos se comprenden por su carácter dinámico y envolvente, ya que articulan los problemas y concepciones básicos de la filosofía ${ }^{9}$.

La paradoja consiste en que, por un lado, el Ministerio de Educación Nacional no clarifica los estándares propios de la filosofía, y los deja a la consideración de cada institución educativa y, sin embargo, por otro lado, el ICFES asume que el estudiante debe manejar conceptos básicos de antropología, ética, política, ontología, epistemología, estética, etc., que han trabajado los grandes filósofos en el desarrollo de sus propias teorías. Por tanto, presupone que el estudiante ha tenido formación filosófica, tanto en la parte histórica, como en el desarrollo de la actitud filosófica.

\footnotetext{
${ }^{9}$ Ministerio de Educación Nacional. Servicio Nacional de Pruebas ICFES. Examen de Estado para el ingreso de la educación superior. Cambios para el Siglo XXI. Sociales y Filosofía. Bogotá, 1.999, p. 53.
} 
¿Cuáles son los criterios en los que se fundamenta el ICFES para evaluar contenidos y competencias de filosofía, si no existe claridad en los estándares básicos para la enseñanza de la filosofía en secundaria? Consideramos que mientras estas ambigüedades y paradojas no se disipen, los profesores de filosofía de secundaria seguiremos caminando sin un rumbo fijo, y sin saber el sentido que justifica nuestro quehacer pedagógico.

El profesor Jorge Aurelio Díaz, en el ya citado curso de la Universidad Nacional, planteó lo siguiente:

“¿Se justifica enseñar filosofía en el bachillerato? Esta pregunta se justifica por varias razones. En primer lugar, si no sabemos por qué hay que enseñar filosofía, no sabremos tampoco qué enseñar bajo el nombre de filosofía, y menos aún cómo hacerlo. Además, la filosofía, más que cualquier otra asignatura, debe estar en condiciones de justificar su presencia, y sólo así podrá establecer su función dentro de un pensum de estudios. Pero, sobre todo, porque si alguna justificación puede tener la filosofía es la de trasmitir un pensamiento crítico, capaz de dar razón de sí, de justificar sus propias credenciales. Pero hay además una razón adicional para plantearse la pregunta inicial: no existe un consenso claro sobre lo que debemos entender bajo el nombre de filosofía, y no creo que podamos esperar conseguirlo en un el futuro cercano. Entonces, ¿Qué debemos hacer? ${ }^{10}$

Para dar respuesta a estos interrogantes, y para disipar las ambigüedades y paradojas del sistema educativo, convocamos a la comunidad filosófica colombiana a reflexionar sobre nuestro propio quehacer, y a emprender una empresa que justifique tanto el sentido como la importancia de la enseñanza de la filosofía en secundaria.

Parece ser que la situación en Colombia es lugar común en otros países latinoamericanos. La comunidad de filósofos del Perú aborda inquietudes similares en la Declaración de Arequipa a favor de la enseñanza de la filosofía en el Perú,

\footnotetext{
${ }^{10}$ Díaz, Jorge Aurelio. ¿Se justifica enseñar filosofía en el bachillerato? En: Curso de Extensión: Enseñanza de la filosofía. Departamento de filosofía, Universidad Nacional de Colombia, septiembre 20 - Diciembre 6 de 2005. (programa) p. 7.
} 
donde abiertamente acuerdan entre otras cosas: "RECOMENDAR a las universidades e instituciones educativas del Perú, así como a la Sociedad Peruana de Filosofía, a fin de que se pronuncien públicamente a favor de la necesidad y urgencia de la filosofía entre los jóvenes peruanos"11.

\section{2. ¿Qué se Enseña Actualmente en Colombia en la Asignatura de Filosofía?}

El vacío dejado por las políticas del Ministerio de Educación Nacional no ha sido obstáculo para que se siga enseñando filosofía en secundaria. Para llevar a cabo esta labor los profesores hemos trazado nuestros propios programas, con el fin de integrar los contenidos de esta asignatura con las inquietudes, experiencias y realidades que envuelven la vida académica, política, social y cotidiana de nuestros estudiantes. En esta búsqueda hemos conquistado logros significativos como seminarios, talleres, foros, paneles, etc., donde los estudiantes han encontrado un espacio para reflexionar, desarrollar la actitud filosófica y compartir sus investigaciones, inquietudes y propuestas.

A pesar de los esfuerzos hechos, consideramos que la enseñanza actual de la filosofía en secundaria enfrenta una serie de dificultades, las cuales abordaremos a la luz de los siguientes interrogantes: ¿Quiénes enseñan? ¿Qué textos apoyan el aprendizaje? ¿Qué se enseña?

\section{1 ¿Quiénes enseñan?}

Dentro de la estructura interna de las instituciones educativas a cada docente se le asignan un número de horas a la semana, regulado por el Ministerio de Educación Nacional: "El tiempo total de la asignación académica semanal de cada docente de educación básica secundaria y educación media, será de veintidós horas efectivas de sesenta minutos, las cuales serán distribuidas por el rector o director en períodos de clase de acuerdo con el plan de estudios"12.

\footnotetext{
11 VI Coloquio Nacional de Filosofía. Arequipa (Perú), 3de diciembre de 2004. (http://www.boulesis.com/docs/sistema-educativo/declaracion-a-favor-filosofia-peru.pdf). Visitada el 17-032007.

${ }^{12}$ Ministerio de Educación Nacional. Decreto No. 1850 agosto 13 de 2002. Artículo 5, parágrafo.
} 
Para poder cumplir esta disposición, los directivos de las instituciones educativas distribuyen la carga académica por áreas de conocimiento y grados. Con respecto a la asignatura filosofía, la problemática señalada se torna más aguda. Al no ser una asignatura obligatoria reglamentada por el Ministerio de Educación Nacional, su intensidad horaria queda a consideración de las directivas de la institución. En la mayoría de casos las instituciones asignan estas horas a un profesor de ciencias sociales para completarle la carga académica. Esto trae como consecuencia que la asignatura de filosofía, por un lado, sea ubicada en el área de ciencias sociales y, por otro lado, no sea enseñada por licenciados en filosofía. Al respecto Vargas Guillen afirma: "Se estima que por cada cien cursos de los que se dictan en este nivel educativo sólo son atendidos tres por un profesional en filosofía". ${ }^{13}$

$\mathrm{Si}$ bien se pueden establecer relaciones interdisciplinarias fructíferas y enriquecedoras entre las ciencias sociales y la filosofía, no podemos confundirlas, ya que, como señala Salazar Bondy, ellas se diferencian tanto en su naturaleza como en su objeto: "La educación científica se diferencia de la filosófica, porque en filosofía no hay un resultado neto y acabado de conocimientos que pueda ser trasmitido, sino que lo enseñable es una actitud, una técnica, un modo de encarar y pensar el cosmos... En cambio en la ciencia la enseñanza se aplica a un sector determinado, a una parcela del conocimiento, de donde resultan asignaturas eminentemente selectivas y especializadas... Hay en la enseñanza filosófica un imperativo de crítica y problematización frente al cual la enseñanza científica parece, en algunos casos, compadecerse con elementos de dogmatismo ${ }^{14}$.

Sin demeritar a nuestros colegas licenciados en ciencias sociales, consideramos que la enseñanza de la filosofía exige unas competencias que escapan a su formación, como es el caso de la actitud filosófica; es decir, no sólo se enseña filosofía, sino que también se enseña a reflexionar filosóficamente.

\footnotetext{
${ }^{13}$ Vargas Guillen, Germán. Espacios de acción de la filosofía en la sociedad. En. Conferencia dada en el foro: ¡Enseñar filosofía!. Bogotá, Universidad Pedagógica Nacional, 2005.

${ }^{14}$ Salazar Bondy, A. Didáctica de la filosofía. Lima, Arica, 1967, p. 21.
} 


\section{2 ¿Qué textos apoyan el aprendizaje filosófico en secundaria?}

En este ítem queremos referirnos al papel que cumplen los textos guía de filosofía en secundaria, pues éstos constituyen una herramienta práctica para nuestro quehacer en el aula. Algunas editoriales se han dado a la tarea de proponer un derrotero para la enseñanza de la filosofía y adaptarlo a los estándares básicos de competencias.

Tomemos como ejemplo el trabajo realizado por la Editorial Santillana, en los textos Filosofía I y Filosofía II. La editorial intenta generar una propuesta acorde con las nuevas políticas del Ministerio de Educación Nacional: la serie Filosofía Santillana para educación media tiene como punto de referencia la Ley 115 General de Educación. Esencialmente la serie busca contribuir al logro de los fines de la educación en Colombia. Para llevar a cabo los contenidos propuestos, la Editorial entrega al docente una guía donde se especifican: núcleos temáticos, unidades didácticas, temas y estándares. Cada núcleo temático se desarrolla bajo la siguiente estructura: esquema conceptual, logros -conceptuales, procedimentales y actitudinales- filosofía y vida cotidiana, sugerencias didácticas $y$ actividades de ampliación ${ }^{15}$. Además los manuales traen fragmentos de obras filosóficas significativas -antología de textos- y una prueba de preparación para el Examen del Estado.

Muchos docentes, ante el vacío dejado por las políticas del Ministerio de Educación Nacional, articulan las propuestas de los textos guía de las distintas editoriales en el diseño de la sábana de contenidos exigidos por las instituciones educativas para el diseño curricular de las distintas asignaturas. Si bien el trabajo de las editoriales es interesante, no deja de ser sólo una propuesta suelta que puede ser acogida o no por los docentes de filosofía en secundaria.

\section{3 ¿Qué se enseña?}

En la actualidad los docentes de filosofía, como ya lo habíamos dicho, tenemos cierta "autonomía" para elaborar el programa que vamos a llevar a cabo en los

\footnotetext{
${ }^{15}$ Cfr. Ibíd. pp. 10-19.
} 
grados décimo y undécimo. La mayoría de docentes, a la hora de especificar los contenidos de la asignatura, nos vemos enfrentados a un dilema: desarrollar la actitud filosófica en nuestros estudiantes desde la historia de la filosofía o desde los ejes temáticos -ontología, antropología, epistemología...-que permiten formar en los estudiantes un pensar crítico y reflexivo sobre la realidad en la que están inmersos.

En el curso de la Universidad Nacional, citado anteriormente, el profesor Gonzalo Serrano se refiere a este problema: "Hay dos posiciones enfrentadas respecto de si el ejercicio filosófico requiere de la familiaridad con los grandes pensadores del pasado, o si por el contrario es menester más bien concentrarse en ciertas destrezas formales para pensar por sí mismo"16.

En los textos guía, tal como lo afirma el profesor Germán Marquinez, también se plantea este dilema: "Hay textos y profesores de filosofía que se limitan al estudio de la historia de la filosofía, y hay otros que se consagran al estudio de temas sin ocuparse de la historia de la filosofía. El estudio de la filosofía a través de sus grandes temas permite descubrir el sentido de la vida, de la realidad, que se nos ofrece cotidianamente como problema. $Y$ su estudio a través de la historia nos brinda la posibilidad de conocer qué y cómo pensaron otras personas, los filósofos, antes que nosotros, sobre los problemas vitales que afectaban a su generación o que siempre han afectado a la humanidad"17.

Dialogando con algunos colegas sobre sus programas y contenidos, percibimos que difícilmente se encuentran dos programas iguales y que, en su gran mayoría, éstos dependen ya sea de la universidad donde se formó, o de la corriente filosófica a que el docente pertenece.

\section{¿Qué nos queda por hacer?}

\section{La filosofía como eje transversal.}

\footnotetext{
${ }^{16}$ Serrano, Gonzalo. El papel de la historia del pensamiento en la enseñanza de la filosofía. En: Curso de Extensión: Enseñanza de la filosofía. Departamento de filosofía, Universidad Nacional de Colombia, septiembre 20 - Diciembre 6 de 2005. (programa) p. 3.

${ }^{17}$ Marquinez, Germán; otros. Filosofía. Perspectiva Latinoamericana 11º. Bogotá, El Búho, 1994, p. 15.
} 
Antes de desarrollar la idea de la importancia de la filosofía como asignatura, reflexionemos, de la mano de Estanislao Zuleta ${ }^{18}$, sobre otra idea de no menor importancia: la filosofía como eje transversal del proceso enseñanza/aprendizaje.

Según Zuleta, el bachillerato es una ensalada extraordinaria de materias diversas que, por una parte, se dan incomunicadas unas de las otras y, por otra parte, no brindan saberes prácticos para la vida del estudiante. Tal como existe, reduce la enseñanza a la transmisión de datos y el aprendizaje a memorización, confunde educación con información y trasmite resultados, sin enseñar procesos de conocimiento. La educación y los maestros nos hicieron un mal favor: nos ahorraron la angustia de pensar ${ }^{19}$.

Además de enseñar resultados sin enseñar procesos de conocimiento, según Zuleta, existe otro problema fundamental: En la escuela se enseña sin filosofía, y ese es el mayor desastre de la educación. Se enseña geografía sin filosofía, biología sin filosofía, historia sin filosofía y filosofía sin filosofía ${ }^{20}$. Esto trae como consecuencia que el estudiante vea el currículo de forma fragmentada y dispersa, y que no articule los saberes de manera integral.

Muchas asignaturas del currículo operan con el plano cartesiano. No obstante ¿cuántos estudiantes comprenden el proceso emprendido por Descartes para llegar a este resultado? Mientras el estudiante no se apropie del proceso que condujo a dicho resultado, el conocimiento puede degenerar en "dogma": mientras uno no sepa por qué menos por menos da mas, no hay apropiación del proceso que conduce a dicho resultado, lo cual demuestra que también las matemáticas pueden se un dogma, al igual que la religión o la historia sagrada ${ }^{21}$.

Para superar esta dificultad, Zuleta considera necesario que el estudiante desarrolle su capacidad de pensar por sí mismo y su capacidad de pensar de manera universal, es decir, que desarrolle la actitud filosófica.

\footnotetext{
${ }^{18}$ Cfr. Valencia, Alberto (compilador). Conversaciones con Estanislao Zuleta. Cali: Fundación Estanislao Zuleta, 1997, pp. 229 - 268.

${ }^{19}$ Ibíd. pp. $233-234$.

${ }^{20}$ Ibíd. p. 236.

${ }^{21}$ Ibíd. p. 233.
} 
perfeccionamiento de estas competencias es precisamente la función propia de la enseñanza de la filosofía en secundaria. Un programa de educación pensado así dificultaría seguramente crear buenos funcionarios, pero probablemente se construiría la posibilidad de formar gentes que luchen por un tipo de sociedad en la que valga la pena vivir y valga la pena estudiar22.

Una vez planteada la importancia de la filosofía como eje transversal y articulador de los distintos saberes del currículo, nos surgen los siguientes interrogantes: ¿cómo promover las competencias necesarias para entender la globalidad del proceso de enseñanza/aprendizaje sin el espacio específico que brinda la asignatura de filosofía? Si la educación media vocacional tiene como fin la comprensión de ideas y valores universales para formar personas que respondan a unas demandas de la sociedad, que contribuyan al mejoramiento y el cambio de la misma ¿por qué la filosofía no es considerada Área obligatoria y fundamental?

\section{La importancia de la asignatura de filosofía en secundaria}

Las políticas de educación en Colombia pretenden que el estudiante desarrolle la capacidad de poner en marcha, de modo integrado, los conocimientos adquiridos (teóricos, prácticos, axiológicos y actitudinales), y los rasgos de su personalidad (competencias) con el fin de que esté en condiciones de resolver situaciones diversas. Es decir, el aprendizaje implica que el estudiante aprenda a: ser, saber, hacer y convivir.

La educación media vocacional (décimo y undécimo) -que es la culminación y consolidación de los niveles escolares anteriores-, tiene como fin la comprensión de las ideas y los valores universales, y la preparación para el ingreso del educando a la educación superior y al trabajo.

Teniendo en cuenta este fin, consideramos que la enseñanza de la filosofía, por su naturaleza universal y por la reflexión que hace en torno al sentido de la vida, abre un horizonte que complementa y enriquece las búsquedas vocacionales en las que se encuentra inserto el estudiante.

\footnotetext{
22 Ibíd. p. 237.
} 
Es claro que el objetivo de los profesores de filosofía en secundaria NO es el de formar filósofos en el sentido estricto de la palabra; éste no es trabajo de la educación media, sino de la educación superior. Nosotros somos compañeros de viaje en el largo camino del asombro, los interrogantes y los problemas que rondan la existencia de nuestros estudiantes; somos los encargados de sembrar en ellos la semilla para que, a través de la actitud filosófica, fortalezcan su personalidad, asuman la responsabilidad de ser sujetos de su propia historia y, de manera autónoma, le den sentido a su existencia.

Según Salazar Bondy el estudiante: "Guiado por el profesor, tiene que superar uno a uno los obstáculos de la conciencia ingenua, primitiva, prejuiciosa o unilateral de lo real, y acceder gradualmente al nivel de un pensar filosófico siquiera elemental"23.

Para despertar la actitud filosófica es de vital importancia que el estudiante, al acercarse al mundo para conocerlo, deje hablar a las cosas mismas de manera objetiva; ello implica que deba partir de sus propias experiencias subjetivas, no con el fin de quedarse en ellas, sino de proyectarlas a un mundo intersubjetivo desde una visión racional y orgánica de la realidad. Despertar la actitud filosófica, requiere que los docentes recurramos a ciertas herramientas y potenciemos ciertas dimensiones tanto cognitivas como afectivas.

Despertar la actitud filosófica es importante, ya que ésta permite, por un lado, problematizar las grandes preguntas de la filosofía y, por otro lado, problematizar la propia existencia del estudiante y su relación con el mundo, los otros y la tradición histórico-cultural.

Una vez planteados los problemas, el docente encuentra el terreno allanado para enriquecer el conocimiento desde panorámicas existenciales, temáticas, históricas o prospectivas. Este ejercicio permite que el estudiante comprenda sus propios problemas a la luz de otros pensadores y en otras épocas. Por ejemplo, a partir de la pregunta por el hombre, podemos plantear reflexiones existenciales: ¿Quién

\footnotetext{
${ }^{23}$ Salazar Bondy, A. Didáctica de la filosofía. Lima, Arica, 1967, p. 31.
} 
soy? ¿Quién es el otro? O temáticas: ¿Qué es el hombre según la antropología? O históricas: ¿Qué han pensado los distintos filósofos en las diversas épocas sobre el hombre? O prospectivas: ¿Qué tipo de hombre quiero ser?

En un contexto vocacional, donde el estudiante está ad portas de tomar decisiones definitivas para su realización personal y profesional, consideramos que las reflexiones filosóficas no son sólo importantes, sino también imprescindibles, ya que lo que está en juego es precisamente uno de los problemas fundamentales de todo quehacer filosófico: el sentido de la vida.

En un mundo donde los juicios de valor sobre la juventud casi siempre son pesimistas, donde la vida juvenil es definida como la era del vacío, del absurdo o del sin-sentido; donde la capacidad de asombro es opacada por un mundo ya dado, prefigurado y predeterminado, donde se padece una crisis de valores; en un mundo tal, la pregunta por el significado de la existencia y la búsqueda de su respuesta siguen justificando la presencia de la asignatura de filosofía en secundaria.

Abordar este camino supone un esfuerzo personal del estudiante, y un buen acompañamiento espiritual del docente. La actitud filosófica puede ser ese faro que alumbra en la oscuridad de la desidia, del inconformismo y del vacío de sentido.

\section{PERSPECTIVAS}

Como lo señalamos desde el principio, el fin de esta investigación no es dar respuestas acabadas, sino abrir un espacio de discusión. Si esto se logra, nos daremos por bien servidos. Por ello creemos que, además de las problemáticas planteadas anteriormente, es pertinente también reflexionar de manera crítica sobre los siguientes asuntos:

\section{A propósito de la propuesta de lineamientos curriculares para filosofía}

El Ministerio de Educación Nacional convocó a un concurso con el fin de elaborar los lineamientos curriculares para la enseñanza y aprendizaje de la filosofía. Un grupo de la Universidad Pontificia Bolivariana fue el escogido y ha realizado 
varios borradores de los lineamientos curriculares para la enseñanza y el aprendizaje de filosofía. Si bien muchas de las temáticas desarrolladas en el Documento nos atañen, consideramos que un análisis profundo y crítico amerita discusiones que desbordan la intención de la presente ponencia. Esperamos que el debate en torno a esta propuesta nos brinde elementos significativos y oriente el quehacer de la asignatura de filosofía en secundaria.

- Al analizar y comparar las propuestas educativas y las Leyes Generales de Educación de algunos países de América Latina, dadas a partir de la década de los noventas, nos queda la impresión de que el perfil de persona que se pretende formar está orientado hacia el desarrollo de dos competencias básicas: las técnicas -saber hacer-y las ciudadanas -saber convivir-. En este contexto, y desde las disciplinas propias de la filosofía, cabe preguntar:

1. Cuestión epistemológica: ¿Dónde queda el desarrollo de las competencias críticas y del saber divergente, propias de la actitud filosófica, en dichas propuestas y leyes?

2. Cuestión antropológica: ¿Cuál es el ideal de hombre hacia el cual se orienta la educación en nuestro país?

3. Cuestión Política: ¿Qué tipo de persona forman las "competencias ciudadanas"?, ¿Para qué tipo de democracia queremos "buenos ciudadanos"?

4. Cuestiones ética y axiológica: ¿Cuáles son los paradigmas de autoridad moral para los jóvenes? ¿Cuáles son los valores que fundamentan las "competencias ciudadanas"?

5. Cuestión estética: ¿Qué referentes estéticos le brinda la cultura actual a los jóvenes?

6. Cuestión ontológica: ¿De dónde venimos? ¿Quiénes somos? ¿Para dónde vamos?

- Vemos con preocupación el movimiento denominado Filosofía para Niños (FpN). Compartimos con esta propuesta la idea de que la filosofía posee un carácter 
articulador que posibilita la generación de pensamientos y conocimientos, no sólo en la educación media vocacional, sino también en preescolar y media básica. Sin embargo, no nos resulta claro su referente epistemológico. ¿La FpN es una adaptación del pragmatismo para los niños? En sentido estricto ¿la FpN puede ser considerada filosofía? Por ello unimos nuestra voz a las afirmaciones de Adela Cortina "Que la educación es tarea clave para el pragmatismo norteamericano es bien conocido, como también que hoy en día camina en este sentido la corriente de «Filosofía para Niños» de M. Lipmann, tan extendida en los países de habla hispana".

También unimos nuestra voz a la del profesor y Germán Vargas Guillen: "Con suspicacia y reticencia dejo como último aspecto de la docencia la llamada filosofía para niños. El proyecto de Lipman y su apropiación nacional en realidad tienen una idea bastante laxa de la filosofía, pero, sin duda, desarrollan estrategias de lectura en los niños. Desarrollar estrategias para que los niños tengan más relación con el texto, con el análisis, con la argumentación, es válido; pero personalmente no alcanzo a pensar que ahí haya filosofía".

- Igualmente vemos con sospecha la propuesta que la Ley General de Educación da a la formación en valores, ética y democracia, a partir del ejemplo. Si bien es una retórica bien planteada, su aplicación resulta difícil en una sociedad donde los referentes de autoridad, tanto moral como política, han caído en descrédito. Según la Ley, el estudiante se forma en estas competencias siguiendo el ejemplo "del ambiente, del comportamiento honesto de directivos, educadores y personal administrativo, de la aplicación recta y justa de las normas de la institución...", pero pasa por alto algunas características del entorno contrarias a dicha formación:

Con respecto al Ambiente, debemos decir que éste se ve significativamente influenciado por los medios de comunicación, los cuales en su mayoría promueven ejemplos y modos de ser que premian al timador 0 al que desarrolla "competencias" para pasar por encima del otro, manipular para su propio beneficio, traicionar por ejemplo los realitys. 
Respecto a los Educadores. Vemos que en la sociedad actual el docente ha dejado de ser paradigma, y la docencia ha perdido su principio fundamental de "vocación". En palabras de algunos estudiantes, un profesor de biología o química es "un médico frustrado", un profesor de dibujo técnico, "un ingeniero en potencia", y un docente de filosofía, "alguien que está casi loco". Según ellos todos somos "pobresores".

En relación a Los directivos tenemos que en pocas instituciones educativas los estudiantes tienen contacto directo con los directivos. ¿Cómo seguir el ejemplo de personas con las que poco se interactúa?

Y en lo que concierne a La aplicación recta y justa de las normas institucionales, sabemos que los manuales de convivencia de los colegios son redactados por abogados especialistas, para defender a la institución de posibles demandas y/o problemas legales. Son, por una parte, netamente coercitivos y correctivos, y, por otra, poco formadores y conciliadores.

Al tematizar la aplicación recta y justa de la norma, las preguntas que constantemente los estudiantes hacen son: ¿Por qué a un político le dan prebendas carcelarias si es un delincuente? ¿Por qué se le puede dar la casa por cárcel? ¿Cuáles son los parámetros que mide la intención del robo? ¿No es más condenable el que roba para el enriquecimiento propio y le hace daño a todo un país que el que roba por hambre y necesidad?

Si el sentido de la cultura apunta sólo hacia las cosas materiales ¿por qué ha de sorprendernos ver jóvenes consumistas? Si el sentido de los medios de comunicación apunta hacia el sexo ¿por qué ha de sorprendernos jóvenes adictos a la pornografía? Si las instituciones sociales (familia, colegio, universidad, etc.) no muestran posibilidades reales de vida auténtica ¿por qué sorprendernos frente a jóvenes deprimidos, viciosos, anarcos o reaccionarios? En la oscuridad, cualquier cosa, lo que esté más a la mano, lo que implique menos esfuerzos, puede servir para orientar la vida. Estos jóvenes le dan sentido a la vida, pero el problema es 
que ese sentido apunta a "algo" que no los realiza como personas en medio de una comunidad intersubjetiva.

El mundo de los jóvenes, parafraseando a Husserl, impone un derrotero a los docentes, directivos, padres de familia, filósofos y gobernantes: ser funcionarios de la humanidad. Esta tarea tiene como fin ser corresponsables, tanto con el legado que heredamos de la tradición, como con el horizonte de posibilidades que dejamos a las presentes y futuras generaciones. El maestro Daniel Herrera al respecto afirma: "La filosofía, gracias a su reflexión crítica y sistemática, no sólo tiene por objeto expresar a nivel conceptual la realidad, sino también el proyectar modelos operativos que posibiliten la transformación de la realidad. Esto se puede comprobar de una manera muy palpable en la reflexión filosófica sobre la praxis política. Todo aquello por lo que se lucha hoy en día en términos políticos: democracia, derechos humanos, reformas constitucionales, etc., es fruto de la reflexión filosófica de pensadores del pasado ${ }^{24}$.

La invitación es entonces a motivar la actitud filosófica en los jóvenes, con la esperanza de que ellos planteen nuevas respuestas a los nuevos problemas; es decir, con la esperanza de que ellos salgan de su minoría de edad con la firme decisión de pensar por sí mismos. La minoría de edad estriba en la incapacidad de servirse del propio entendimiento, sin la dirección de otro. Uno mismo es culpable de esta minoría de edad cuando la causa de ella no yace en un defecto del entendimiento, sino en la falta de decisión y ánimo para servirse con independencia de él, sin la conducción de otro. ¡Sápere aude! ¡Ten valor de servirte de tu propio entendimiento! ${ }^{25}$.

\footnotetext{
${ }^{24}$ Herrera Restrepo, Daniel. ¿Qué significa ser filósofo? en: Folios. Segunda época. Segundo semestre de 2005. No. 22 Bogotá: Universidad Pedagógica Nacional. p. 7.

${ }^{25}$ Kant, E. ¿Qué es Ilustración? En: Filosofía de la Historia, Buenos Aires, Nova, 1964. p. 1
} 